\title{
Hepatic stem/progenitor cells and stem-cell transplantation for the treatment of liver disease
}

\author{
Sei Kakinuma ${ }^{1,2}$, Hiromitsu Nakauchi ${ }^{1,2}$, and Mamoru Watanabe ${ }^{3}$ \\ ${ }^{1}$ Division of Stem Cell Therapy, Center for Stem Cell and Regenerative Medicine, The Institute of Medical Science, University of Tokyo, \\ 4-6-1 Shirokanedai, Minato-ku, Tokyo 108-8639, Japan \\ ${ }^{2}$ JST, ERATO, Nakauchi Stem Cell and Organ Regeneration Project, Tokyo, Japan \\ ${ }^{3}$ Department of Gastroenterology and Hepatology, Tokyo Medical and Dental University, Tokyo, Japan
}

\begin{abstract}
Allogeneic liver transplantation is still the only effective treatment available to patients with liver failure. However, because there is a serious shortage of liver donors, an alternative therapeutic approach is needed. Transplantation of mature hepatocytes has been evaluated in clinical trials, but the long-term efficacy remains unclear and the paucity of donor cells limits this strategy. Stem-cell transplantation is a more promising alternative approach. Several studies have provided information about the mechanism underlying the proliferation and differentiation of hepatic stem/progenitor cells. Moreover, in experimental models of liver disease, transplantation of hepatic stem/progenitor cells or hepatocyte-like cells derived from multipotent stem cells led to donor cell-mediated repopulation of the liver and improved survival rates. However, before stem-cell transplantation can be applied in the clinic to treat liver failure in humans, it will be necessary to overcome several difficulties associated with the technique.
\end{abstract}

Key words: hepatocyte transplantation, hierarchy, single-cell based analysis

\section{Introduction}

Stem cells have been identified in a variety of tissues, where they play critical roles in the tissues' maintenance and repair. Identification and characterization of hepatic stem cells has been technically difficult, however, because sensitive and stable single-cell-based assays have not yet been developed for the liver. Nonetheless, hepatic stem cells were recently identified in murine fetal liver using prospective single-cell-based methods,

Received / Accepted: September 5, 2008

Reprint requests to: S. Kakinuma after which several groups reported the availability of hepatic stem/progenitor cells for the treatment of liver diseases, as well as hepatocyte-like cells derived from pluripotent stem cells. Here we focus on the biological characteristics of hepatic stem/progenitor cells and discuss the experimental evidence supporting the efficacy of stem-cell transplantation for the treatment of liver disease.

\section{Stem cells and terminal differentiation}

Stem cells are generally defined as cells exhibiting two properties: a capacity for self-renewal and potency for multilineage differentiation. ${ }^{1}$ They are the source of progenitor cells committed to one or several lineages. The committed progenitor cells exhibit a capacity for active proliferation and supply abundant daughter cells, which in turn give rise to terminally differentiated cells.

Stem cells are classified into three categories: totipotent, pluripotent, or multilineage somatic. Totipotent stem cells are capable of generating a fetus in utero by themselves over the course of ontogeny (e.g., a fertilized egg is a totipotent cell). Stem cells lacking potency for ontogeny are excluded from this category. Embryonic stem (ES) cells are capable of differentiating into cells derived from any of the germ layers, including germ line cells; however, they cannot generate a fetus without an interaction between themselves and a fertilized egg. For example, injection of ES cells into a mouse blastocyst leads to generation of a chimeric mouse comprised of ES cells and host cells. Thus, ES cells are classified as pluripotent. Induced pluripotent stem cells (iPS cells) exhibit characteristics similar to those of ES cells. ${ }^{2}$ Another example of pluripotent stem cells is mesenchymal stem cells (MSCs). These are a type of adult stem cell that can be easily isolated from a variety tissues and expanded in vitro, ${ }^{3}$ and are capable of differentia- 


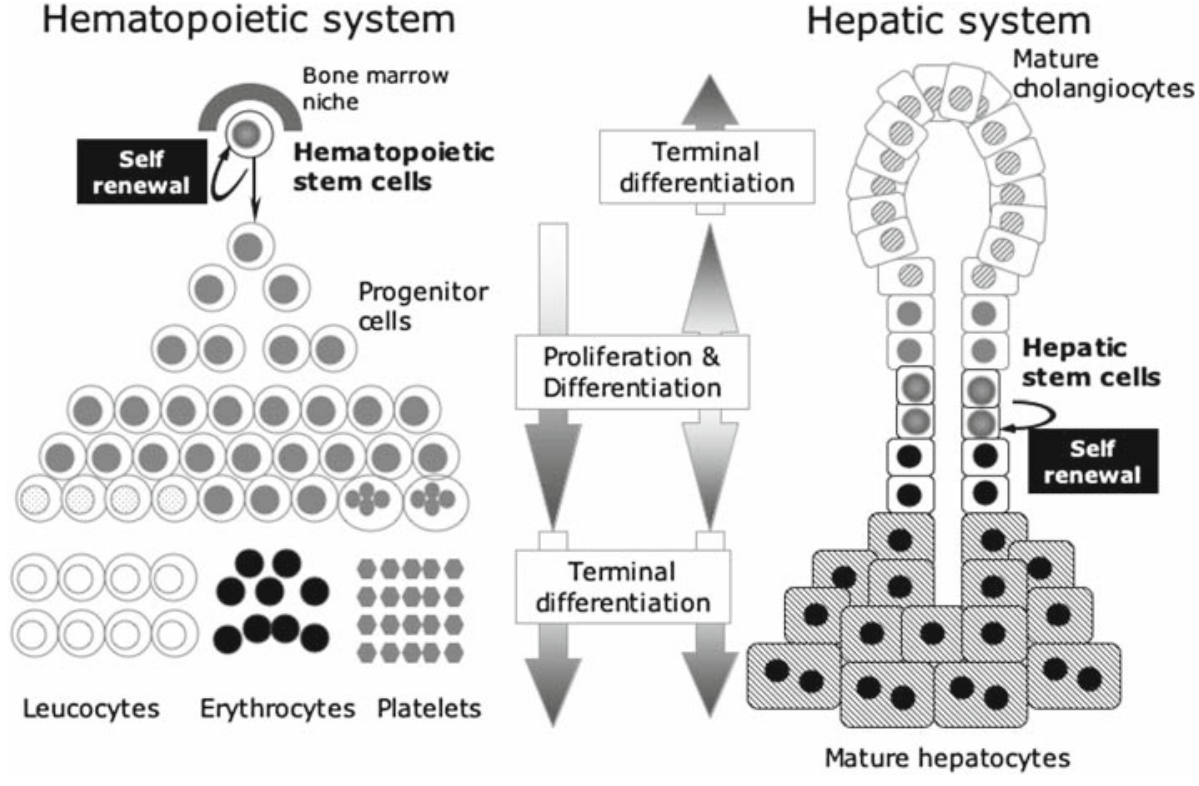

Fig. 1. Hierarchy of stem cells, progenitors, and differentiated cells. Left panel shows the stem-cell system in hematopoiesis. Right panel shows the proposed model of the liver stemcell system tion not only into mesodermal cells (e.g., adipocytes, myocytes, chondrocytes, and osteocytes) but also into both endodermal and ectodermal (e.g., neuroglia) cells, ${ }^{4,5}$ which is indicative of their pluripotency.

Somatic stem cells, by contrast, do not generally exhibit a capacity to differentiate into any cell type other than their own tissue. The best-studied somatic stem cell is the hematopoietic stem cell (HSC). HSCs play a major role in the maintenance of hematopoietic homeostasis, exhibit a capability for self-renewal, and generate progenitor cells. ${ }^{6}$ The progenitor cells in turn actively proliferate, commit to a particular lineage, and produce abundant daughter cells that differentiate into all types of hematopoietic cells, including granulocytes, monocytes, lymphocytes, dendritic cells, erythrocytes, and platelets (Fig. 1, left panel). Once terminally differentiated, hematopoietic cells generally lose the ability to commit to other cell lineages. Several HSC phenotypes have been characterized. For instance, murine HSCs are enriched in the CD $34^{-} \mathrm{c}-\mathrm{Kit}^{+} \mathrm{Sca}-1^{+}$Lineage fraction in bone marrow, and transplantation of a single cell from this fraction is able to reconstitute lethally irradiated bone marrow over the long-term. ${ }^{7}$ In contrast to ES cells, HSCs in adult bone marrow "hibernate" in response to niche signals under physiological conditions. ${ }^{8}$ Analysis of bromodeoxyuridine incorporation into murine HSCs revealed that all HSCs are regularly recruited into the cell cycle such that, on average, $99 \%$ of HSCs will divide within a period of 57 days, though approximately $75 \%$ of HSCs are quiescent in G0 at any given time. ${ }^{9}$ This capacity to enter and leave a hibernation-like state is of critical biological importance, as it prevents premature HSC exhaustion under conditions of hematopoietic stress. The developmental pathway followed during hematopoiesis has been well studied, and has contributed a great deal to our understanding of the pathogenesis and treatment of leukemia. ${ }^{10}$ Similar analyses of the phenotypes, developmental pathways, and molecular mechanisms underlying the proliferation and differentiation of hepatic stem cells should also contribute to our understanding of hepatocellular carcinoma, and on a conceptual level further our knowledge of cancer stem cells in liver.

\section{Identification of fetal hepatic stem cells}

Somatic stem cells in liver (i.e., hepatic stem cells) are defined as cells capable of self-renewal and terminal differentiation into multiple lineages. Liver lobules are constituted of a variety of cells, including hepatocytes, cholangiocytes, hepatic stellate cells (Ito cells), Kupffer cells, sinusoidal endothelial cells, and Pit cells, among others. Both hepatocytes and cholangiocytes are derived from hepatoblasts in fetal liver, ${ }^{11}$ and Suzuki et al. ${ }^{12}$ showed that a single cell in the c-Met ${ }^{+} \mathrm{CD} 49 \mathrm{f}^{+/ \text {low }} \mathrm{c}-\mathrm{Kit}^{-}$ CD $45^{-}$Ter $119^{-}$fraction from midgestational fetal liver has the capacity for self-renewal in vitro and for bipotential differentiation, indicating that this defined fraction contains hepatic stem cells. Hepatic progenitor cells are also capable of bipotential differentiation and active proliferation, but lack the capacity for selfrenewal. Hepatic stem/progenitor cells can be enriched in mouse fetal hepatic cells based on several cell surface markers, including c-Met, ${ }^{12}$ Dlk, ${ }^{13}$ E-cadherin, ${ }^{14}$ and Liv2. ${ }^{15}$ Rat Dlk ${ }^{+}$cells isolated from midgestational fetal liver exhibit characteristics expected for hepatic stem/ progenitor cells, ${ }^{16}$ but further purification of stem cells 
from the hepatic stem/progenitor-cell fraction has been difficult so far.

Several molecular mechanisms regulating the proliferation and differentiation of hepatic stem/progenitor cells have been described. Our group, for example, has shown that Prospero-related homeobox 1 (Prox1) and liver receptor homolog 1 (Lrh1) regulate proliferation of hepatic stem/progenitor cells. ${ }^{17}$ In that study, overexpression of Prox1 induced migration and proliferation of hepatic stem/progenitor cells, and Prox1 suppressed the expression of $\mathrm{p} 16^{\text {Ink4a }}$, a cdk inhibitor, through regulation of $\mathrm{p} 16^{\mathrm{Ink4a}}$ promoter activity. That proliferation of hepatic stem/progenitor cells overexpressing Prox1 was inhibited by overexpression of Lrh1 suggests that Prox1 and Lrh1 act cooperatively to regulate proliferation of fetal hepatic stem/progenitor cells. Other groups have shown that hepatocyte growth factor signaling induces differentiation of fetal hepatic stem cells, and that the expression of CCAAT/enhancer binding protein $(\mathrm{C} /$ EBP), a basic leucine zipper transcription factor, is dramatically altered during the differentiation step. When C/EBP activity in stem cells is suppressed, they stop differentiating into hepatocyte-lineage cells and proliferate actively. ${ }^{18}$ On the other hand, Tbx3, one of the T-box genes, controls the fate of fetal hepatic stem/ progenitor cells by suppressing $19^{\text {Arf }}$ expression. ${ }^{19}$ As a result, Tbx3-deficient hepatic stem/progenitor cells exhibit cholangiocytic lineage segregation.

\section{Adult hepatic stem/progenitor cells}

How hepatic stem cells in adult liver contribute to the maintenance of differentiated hepatocytes and cholangiocytes remains unclear. This is because adult hepatic stem cells are scarcely detectable under physiological conditions and during the normal process of liver regeneration, presumably because of their small numbers. It has been proposed that "oval cells" may actually be hepatic stem/progenitor cells in the damaged liver. Oval cells are detected after treatments that inhibit the proliferation of intrinsic hepatocytes. For example, they are seen following administration of 2-acetylaminofluorene with partial hepatectomy $(\mathrm{PH})$ to rats $^{20}$ or after providing a diet deficient in 3,5-diethoxycarbonyl-1,4dihydrocollidine (DDC) to mice. ${ }^{21}$ Oval cells express both hepatic (albumin and $\alpha$-fetoprotein) and cholangiocytic (CK-7 and CK-19) markers, ${ }^{22}$ as well as several stem-cell-related markers, including c-Kit, ${ }^{23} \mathrm{CD} 34,{ }^{24}$ and $\mathrm{LIF}^{25}$ Analyses of oval cells have raised the possibility that adult hepatic stem cells are present in the "canals of Herring," and that oval cells originate from the stem cells and differentiate into both the hepatic and cholangiocytic lineages (Fig. 1, right panel). However, because oval cells are scarce in the normal adult liver, it remains unclear whether they differentiate into functional hepatocytes and play a meaningful role in the reconstitution of liver lobules. It is also difficult to identify canals of Herring in normal healthy liver. Consequently, attempts to detect hepatic stem cells in adult normal liver are still underway.

Azuma et $\mathrm{al}^{26}$ showed that normal murine liverderived cells in the nonparenchymal cell fraction isolated through cell aggregate formation contained bipotential progenitor cells. In addition, colony formation assays using rat adult liver cells revealed that $0.043 \%$ of the nonparenchymal cell fraction formed bipotential colonies, and that the frequency of such proliferative colonies did not significantly differ, whether the cells were obtained from normal intact liver or liver after $\mathrm{PH}^{27}$ This suggests hepatic stem/progenitor cells are indeed present in normal adult liver. Recently, it has been reported that epithelial cell adhesion molecule is expressed in nonpathological human livers on hepatic progenitors in the livers of both fetal and adult donors, and that the hepatic progenitors give rise to the hepatocytic and biliary lineages. ${ }^{28}$ As with hematopoietic stem cells, the phenotype of adult hepatic stem cells has not been fully characterized, however. Future establishment of a system for cell isolation, single-cellbased analysis, and transplantation would contribute greatly to the further characterization of the phenotypes of adult hepatic stem cells, as well as to their localization and to the identification of the molecular mechanisms underlying self-renewal and determination of cell fate.

\section{Trial of stem cell therapy for liver diseases}

Liver transplantation is currently the only successful treatment for acute hepatic failure or endstage liver disease. At present, however, a serious donor shortage is a major limitation to its use. Hepatocyte transplantation has the potential to solve this problem. Several studies using rat models of primary hepatocyte transplantation revealed that transplantation leads to efficacious donor chimerism that can rescue animals from lethal hepatic failure ${ }^{29-31}$ Immortalized hepatocytes have also been shown to improve the survival rate in an acute liver failure model. ${ }^{32}$

In two clinical trials, hepatocyte transplantation was shown to be effective for the treatment of liver-based congenital metabolic disorders. Fox et al..$^{33}$ transplanted allogeneic hepatocytes into the liver of a patient with Crigler-Najjar syndrome type I. The patient survived for 11 months, and the patient's hyperbilirubinemia was partially corrected. In addition, Dhawan et al. ${ }^{34}$ reported that transplantation of cryopreserved hepatocytes into patients with inherited factor VII deficiency led in a 
gradual reduction in their requirement for recombinant factor VIIa until, after 8 to 10 weeks, they were receiving only about $20 \%$ of their original dose. It remains unclear, however, whether hepatocyte transplantation can contribute to the rescue of liver-based metabolic diseases over the long-term. Moreover, in vitro expansion of mature hepatocytes is not feasible because longterm cultivation of hepatocytes results in hypofunction of hepatocytic metabolism. It would thus be greatly beneficial if we could generate functional hepatocytes from stem cells, which could be expanded in vitro.

Recent studies have also shown that transplantation of stem cell-derived cells can improve liver function and the survival rate in liver diseases. Initially, ES-cell derived cells could be a source for alternative donor cells. For instance, hepatocyte-like cells have been induced from murine ES cells through embryoid body formation. ${ }^{35,36}$ When murine ES cell-derived hepatocytes enriched through the use of several surface markers were transplanted into injured mouse liver, liver function subsequently improved. ${ }^{37}$ Moreover, Teratani et al. ${ }^{38}$ identified growth factors that enable direct hepatic fate-specification from murine ES cells in monolayer cultures without the necessity of embryoid body formation. Transplantation of ES cell-derived hepatic cells significantly suppressed the onset of fibrosis in mice and improved the survival rate among the recipient mice. ${ }^{38}$ In addition, Cai et al. ${ }^{39}$ described a method for directing the differentiation of human ES cells into hepatic cells in serum-free medium and showed that the transplanted cells were integrated into mouse liver, where they expressed human $\alpha 1$ antitrypsin for at least 2 months. Despite these successful animal studies, there have been no clinical trials using human ES cells to treat liver diseases in human patients because utilization of human ES cells raises serious ethical questions in a lot of countries. In the future, autologous iPS cells could replace human ES cells as a source of donor cells.

MSC-derived hepatocyte-like cells are another potential alternative source of donor cells. Transplantation assays with murine MSCs revealed the presence of donor-derived hepatocytes in recipient livers. ${ }^{40}$ After transplantation of human MSC-derived hepatocytes into nude mice with acute liver failure, donor cells restored such liver functions as ammonia and purine metabolism. ${ }^{41}$ Ideally, MSCs for transplantation would be established from the bone marrow or adipose tissue of the patients themselves. Such autologous MSC transplantation would avoid the problem of immunorejection of the cells. On the other hand, van Poll et al. ${ }^{42}$ showed that systemic infusion of soluble factors secreted from MSCs provided a survival benefit and prevented the release of liver injury biomarkers. This raises the possibility that the transplanted MSCs did not play the crucial role in reconstitution of the recipient liver; instead, the soluble factors that they secreted were protective against liver injury. Thus, administration of the soluble factors secreted from MSCs may be an alternative treatment. Further studies are needed to address this issue.

Hepatic stem cells are also good candidate donor cells for the treatment of liver diseases. Because they are specified to liver, functional differentiation of hepatic stem cells would be technically easier than differentiation of ES/iPS cells. Transplanted rat fetal liver epithelial progenitor cells were able to repopulate a recipient liver subjected to $\mathrm{PH}$, alone or with retrorsine, in syngeneic dipeptidyl peptidase IV (DPPIV) mutant rats. ${ }^{43}$ Likewise, $\mathrm{Dlk}^{+}$hepatic stem/progenitor cells purified from rat midgestational fetal liver were able to extensively repopulate the host liver in syngeneic DPPIV mutant rats subjected to $\mathrm{PH}$ alone. ${ }^{16}$ Thus, fetal hepatic stem/progenitor cells exhibit potency for reconstitution of adult liver under a particular set of conditions. In addition, Wang et al. ${ }^{21}$ reported that transplantation of murine oval cells induced by a DDC diet can repopulate the recipient liver in fumarylacetoacetate hydrolase-deficient mice, and rescue the phenotype. It remains unclear, however, whether adult hepatic stem or progenitor cells, like oval cells, can reconstitute recipient livers not subjected to genetic modification. It will be essential to establish the methodology for therapeutic transplantation of hepatic stem cells to reconstitute the recipient liver using only minimal pretreatment and without genetic modification of the recipients.

\section{Conclusion}

Recently, Takahashi et al. ${ }^{44}$ at Kyoto University reported that pluripotent stem cells can be generated from adult human fibroblasts, thereby establishing human iPS cells. This finding could dramatically change the therapeutic strategy for development of cell-based therapies for lethal liver diseases in the near future, as iPS cells derived from the patients themselves could be utilized as the donor cell source. On the other hand, the methodology for induction of hepatocytes from human iPS cells and elimination of immature undifferentiated cells has not yet been established. Moreover, it remains unclear how hepatic stem/progenitor cells derived from iPS cells should be transplanted so that recipients obtain successful liver repopulation and long-term engraftment. Once we overcome these technical problems, we will be able to utilize a variety of stem cells, including autologous iPS cells, MSCs, and endogenous hepatic stem/progenitor cells, as resources for transplantation on a case-by-case basis (Fig. 2). 


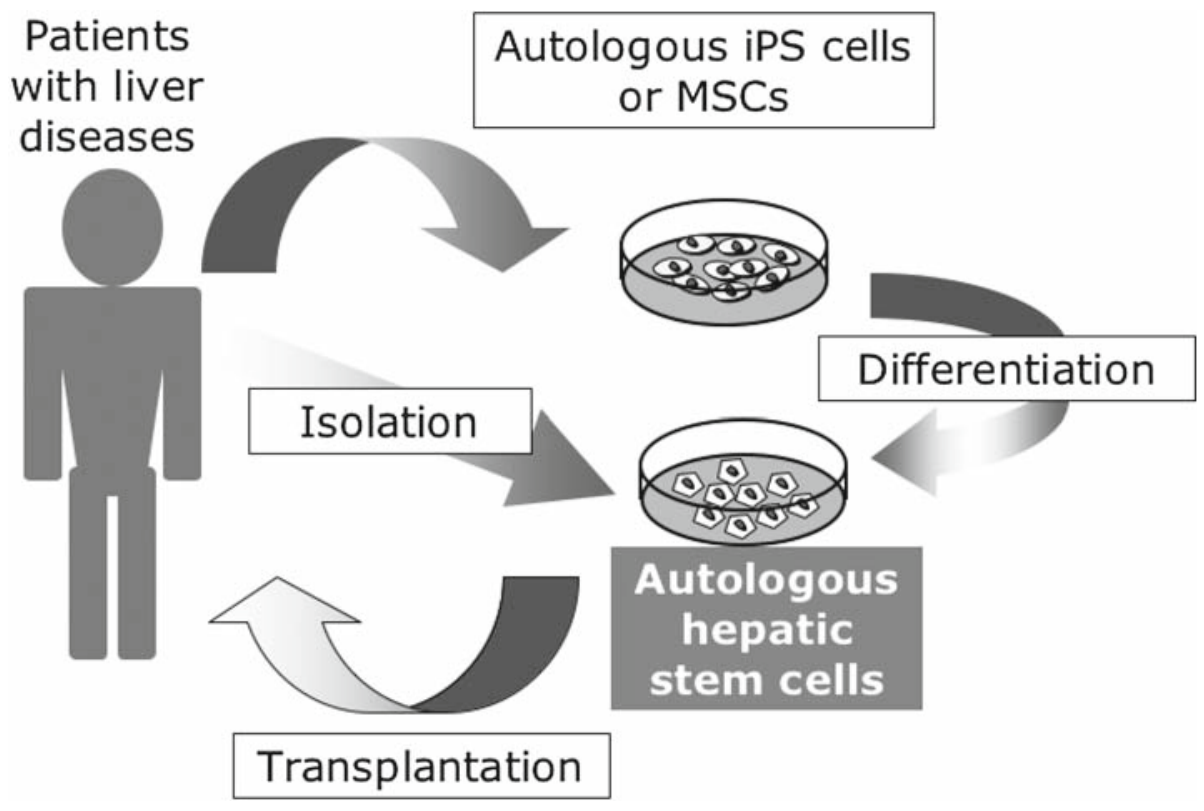

Fig. 2. Stem cell therapy in the future. iPS cells, induced pluripotent stem cells; $M S C s$, mesenchymal stem cells
Acknowledgments. We would like to thank to Dr. Akihide Kamiya of the Institute of Medical Science, University of Tokyo, and Dr. Naoya Sakamoto of the Tokyo Medical and Dental University for the helpful discussions.

\section{References}

1. Reya T, Morrison SJ, Clarke MF, Weissman IL. Stem cells, cancer, and cancer stem cells. Nature 2001;414:105-11.

2. Takahashi K, Yamanaka S. Induction of pluripotent stem cells from mouse embryonic and adult fibroblast cultures by defined factors. Cell 2006;126:663-76.

3. Wagner W, Ho AD. Mesenchymal stem cell preparationscomparing apples and oranges. Stem Cell Rev 2007;3:239-48.

4. Jiang Y, Jahagirdar BN, Reinhardt RL, Schwartz RE, Keene CD, Ortiz-Gonzalez XR, et al. Pluripotency of mesenchymal stem cells derived from adult marrow. Nature 2002;418:41-9.

5. Lee OK, Kuo TK, Chen WM, Lee KD, Hsieh SL, Chen TH. Isolation of multipotent mesenchymal stem cells from umbilical cord blood. Blood 2004;103:1669-75.

6. Ema H, Nakauchi H. Self-renewal and lineage restriction of hematopoietic stem cells. Curr Opin Genet Dev 2003;13:508-12.

7. Osawa M, Hanada K, Hamada H, Nakauchi H. Long-term lymphohematopoietic reconstitution by a single CD34-low/negative hematopoietic stem cell. Science 1996;273:242-5.

8. Yamazaki S, Iwama A, Takayanagi S, Morita Y, Eto K, Ema H, et al. Cytokine signals modulated via lipid rafts mimic niche signals and induce hibernation in hematopoietic stem cells. EMBO J 2006;25:3515-23.

9. Cheshier SH, Morrison SJ, Liao X, Weissman IL. In vivo proliferation and cell cycle kinetics of long-term self-renewing hematopoietic stem cells. Proc Natl Acad Sci U S A 1999;96: 3120-5.

10. Lapidot T, Sirard C, Vormoor J, Murdoch B, Hoang T, Caceres-Cortes J, et al. A cell initiating human acute myeloid leukemia after transplantation into SCID mice. Nature 1994;367: 645-8.

11. Shiojiri N, Lemire JM, Fausto N. Cell lineages and oval cell progenitors in rat liver development. Cancer Res 1991;51:2611-20.
12. Suzuki A, Zheng YW, Kaneko S, Onodera M, Fukao K, Nakauchi $\mathrm{H}$, et al. Clonal identification and characterization of selfrenewing pluripotent stem cells in the developing liver. J Cell Biol 2002;156:173-84.

13. Tanimizu N, Nishikawa M, Saito H, Tsujimura T, Miyajima A. Isolation of hepatoblasts based on the expression of Dlk/Pref-1. J Cell Sci 2003;116:1775-86.

14. Nitou M, Sugiyama Y, Ishikawa K, Shiojiri N. Purification of fetal mouse hepatoblasts by magnetic beads coated with monoclonal anti-e-cadherin antibodies and their in vitro culture. Exp Cell Res 2002;279:330-43.

15. Watanabe T, Nakagawa K, Ohata S, Kitagawa D, Nishitai G, Seo J, et al. SEK1/MKK4-mediated SAPK/JNK signaling participates in embryonic hepatoblast proliferation via a pathway different from NF-kappaB-induced anti-apoptosis. Dev Biol 2002;250: 332-47.

16. Oertel M, Menthena A, Chen YQ, Teisner B, Jensen CH, Shafritz DA. Purification of fetal liver stem/progenitor cells containing all the repopulation potential for normal adult rat liver. Gastroenterology 2008;134:823-32.

17. Kamiya A, Kakinuma S, Onodera M, Miyajima A, Nakauchi H. Prospero-related homeobox 1 and liver receptor homolog 1 coordinately regulate long-term proliferation of murine fetal hepatoblasts. Hepatology 2008;48:252-64.

18. Suzuki A, Iwama A, Miyashita H, Nakauchi H, Taniguchi H. Role for growth factors and extracellular matrix in controlling differentiation of prospectively isolated hepatic stem cells. Development 2003;130:2513-24.

19. Suzuki A, Sekiya S, Buscher D, Izpisua Belmonte JC, Taniguchi $\mathrm{H}$. Tbx3 controls the fate of hepatic progenitor cells in liver development by suppressing p19ARF expression. Development 2008;135:1589-95.

20. Solt DB, Medline A, Farber E. Rapid emergence of carcinogeninduced hyperplastic lesions in a new model for the sequential analysis of liver carcinogenesis. Am J Pathol 1977;88:595-618.

21. Wang X, Foster M, Al-Dhalimy M, Lagasse E, Finegold M, Grompe M. The origin and liver repopulating capacity of murine oval cells. Proc Natl Acad Sci U S A 2003;100 Suppl 1:11881-8.

22. Oertel M, Shafritz DA. Stem cells, cell transplantation and liver repopulation. Biochim Biophys Acta 2008;1782:61-74.

23. Fujio K, Evarts RP, Hu Z, Marsden ER, Thorgeirsson SS. Expression of stem cell factor and its receptor, c-kit, during liver regen- 
eration from putative stem cells in adult rat. Lab Invest 1994;70: 511-6.

24. Omori N, Omori M, Evarts RP, Teramoto T, Miller MJ, Hoang $\mathrm{TN}$, et al. Partial cloning of rat CD34 cDNA and expression during stem cell-dependent liver regeneration in the adult rat. Hepatology 1997;26:720-7.

25. Omori N, Evarts RP, Omori M, Hu Z, Marsden ER, Thorgeirsson SS. Expression of leukemia inhibitory factor and its receptor during liver regeneration in the adult rat. Lab Invest 1996;75: $15-24$.

26. Azuma H, Hirose T, Fujii H, Oe S, Yasuchika K, Fujikawa T, et al. Enrichment of hepatic progenitor cells from adult mouse liver. Hepatology 2003;37:1385-94.

27. Yamamoto H, Togo S, Zheng YW, Kubota T, Taniguchi H, Shimada H. Adult rat hepatic bipotent progenitor cells remain dormant even after extensive hepatectomy. Wound Repair Regen 2007:15:422-9.

28. Schmelzer E, Reid LM. EpCAM expression in normal, nonpathological tissues. Front Biosci 2008;13:3096-100.

29. Makowka L, Rotstein LE, Falk RE, Falk JA, Nossal NA, Langer $\mathrm{B}$, et al. Allogeneic and xenogeneic hepatocyte transplantation in experimental hepatic failure. Transplantation 1980;30:429-35.

30. Rajvanshi P, Kerr A, Bhargava KK, Burk RD, Gupta S. Studies of liver repopulation using the dipeptidyl peptidase IV-deficient rat and other rodent recipients: cell size and structure relationships regulate capacity for increased transplanted hepatocyte mass in the liver lobule. Hepatology 1996;23:482-96.

31. Gagandeep S, Rajvanshi P, Sokhi RP, Slehria S, Palestro CJ, Bhargava KK, et al. Transplanted hepatocytes engraft, survive, and proliferate in the liver of rats with carbon tetrachlorideinduced cirrhosis. J Pathol 2000;191:78-85.

32. Kobayashi N, Fujiwara T, Westerman KA, Inoue Y, Sakaguchi $\mathrm{M}$, Noguchi $\mathrm{H}$, et al. Prevention of acute liver failure in rats with reversibly immortalized human hepatocytes. Science 2000;287: 1258-62.

33. Fox IJ, Chowdhury JR, Kaufman SS, Goertzen TC, Chowdhury NR, Warkentin PI, et al. Treatment of the Crigler-Najjar syndrome type I with hepatocyte transplantation. N Engl J Med 1998;338:1422-6.
34. Dhawan A, Mitry RR, Hughes RD, Lehec S, Terry C, Bansal S, et al. Hepatocyte transplantation for inherited factor VII deficiency. Transplantation 2004;78:1812-4.

35. Hamazaki T, Iiboshi Y, Oka M, Papst PJ, Meacham AM, Zon LI, et al. Hepatic maturation in differentiating embryonic stem cells in vitro. FEBS Lett 2001;497:15-9.

36. Chinzei R, Tanaka Y, Shimizu-Saito K, Hara Y, Kakinuma S, Watanabe M, et al. Embryoid-body cells derived from a mouse embryonic stem cell line show differentiation into functional hepatocytes. Hepatology 2002;36:22-9.

37. Kumashiro Y, Asahina K, Ozeki R, Shimizu-Saito K, Tanaka Y, Kida Y, et al. Enrichment of hepatocytes differentiated from mouse embryonic stem cells as a transplantable source. Transplantation 2005;79:550-7.

38. Teratani T, Yamamoto H, Aoyagi K, Sasaki H, Asari A, Quinn $\mathrm{G}$, et al. Direct hepatic fate specification from mouse embryonic stem cells. Hepatology 2005;41:836-46.

39. Cai J, Zhao Y, Liu Y, Ye F, Song Z, Qin H, et al. Directed differentiation of human embryonic stem cells into functional hepatic cells. Hepatology 2007;45:1229-39.

40. Anjos-Afonso F, Siapati EK, Bonnet D. In vivo contribution of murine mesenchymal stem cells into multiple cell-types under minimal damage conditions. J Cell Sci 2004;117:5655-64.

41. Banas A, Teratani T, Yamamoto Y, Tokuhara M, Takeshita F, Osaki M, et al. Rapid hepatic fate specification of adipose-derived stem cells and their therapeutic potential for liver failure. J Gastroenterol Hepatol 2008 Jun 25 [Epub ahead of print].

42. van Poll D, Parekkadan B, Cho CH, Berthiaume F, Nahmias Y, Tilles AW, et al. Mesenchymal stem cell-derived molecules directly modulate hepatocellular death and regeneration in vitro and in vivo. Hepatology 2008;47:1634-43.

43. Sandhu JS, Petkov PM, Dabeva MD, Shafritz DA. Stem cell properties and repopulation of the rat liver by fetal liver epithelial progenitor cells. Am J Pathol 2001;159:1323-34.

44. Takahashi K, Tanabe K, Ohnuki M, Narita M, Ichisaka T, Tomoda $\mathrm{K}$, et al. Induction of pluripotent stem cells from adult human fibroblasts by defined factors. Cell 2007;131:861-72. 